\title{
Surface electromyographic activity of the erector spinae and multifidus during arm and leg ergometer exercises in young healthy men
}

\section{Daichi Shima}

Wakayama Medical University

Yoshi-ichiro Kamijo ( $\nabla$ yoshikmj@gmail.com )

Dokkyo Medical University Saitama Medical Center

\section{Takamasa Hashizaki}

Wakayama Medical University Hospital

\section{Yuta Minoshima \\ Tatsuya Yoshikawa \\ Wakayama Medical University \\ Yukio Mikami \\ Wakayama Medical University \\ Hideki Arakawa \\ University of Miyazaki \\ Yasunori Umemoto \\ Wakayama Medical University \\ Fumihiro Tajima \\ Wakayama Medical University}

Wakayama Medical University Hospital

\section{Research Article}

Keywords: exercise therapy, rehabilitation, metabolism, arm-crank exercise, cycling exercise

Posted Date: August 3rd, 2021

DOl: https://doi.org/10.21203/rs.3.rs-744450/v1

License: (c) (i) This work is licensed under a Creative Commons Attribution 4.0 International License.

Read Full License 


\section{Abstract}

Surface electromyographic activities of the erector spinae and multifidus during graded arm- and legergometer exercise were investigated. Fifteen young healthy male participants performed arm- and legergometer exercises at $50 \mathrm{~W}$ and $100 \mathrm{~W}$ for $1 \mathrm{~min}$, while monitoring the electrocardiograms of the paraspinal muscles and heart rate, and the root mean squares of the electromyograms were calculated. Time series of contractions of the paraspinal and extremity muscles during both exercises were assessed $(n=7)$. Both paraspinal muscle activities increased with increased workload in both exercises similarly $(P$ $<0.01$, each). Heart rate increased with increased workload, and the increase was greater for armergometer exercise than for leg-ergometer exercise. Each contraction time of trunk and limb muscles suggested that the paraspinal muscles facilitated trunk rotation and prevented excessive lateral bending of the trunk, respectively. The activities of these paraspinal muscles increased with increased workload similarly in both exercises, although heart rate response was different between them.

\section{Introduction}

The paraspinal muscles act to correct any displacement of gravity center in upright posture, and thus contributes in prevention of body falls [1-2]. The paraspinal muscle is also activated in advance of voluntary movements of the arms and legs to maintain body balance, allowing for movement of the arms and legs [3-6].

Although ergometer exercise is frequently used in rehabilitation therapy, since it is known to increase maximal oxygen uptake and muscle strength, lower systolic and diastolic blood pressures, reduce body weight and \%body fat, and improve cognitive function [7-12], information of effects on the paraspinal muscles was still limited. As demonstrated previously, fatigue of the erector spinae occurs after 10 minutes of leg-ergometer exercise [13]. Recording of muscle activity with surface electromyogram (sEMG) demonstrated active contraction of the erector spinae during arm- and leg-ergometer exercises [13-14]. Other studies demonstrated increased activities of the erector spinae and multifidus with increased workload during elbow flexion at 25-40\% maximal voluntary contraction (MVC) [15], squatting at 65$95 \%$ of MVC [16], and $0 \%$ and $20 \%$ of body weight [17]. Thus, the arm- and leg-ergometer exercises can activate the erector spinae and multifidus in a manner dependent on the workload.

Patients with Parkinson's disease suffer from impaired postural control in part due to atrophy of the paraspinal muscles [18-20]. Ergometer exercise is safe and effective for the patients, as recommended in the ACSM's Guidelines [21]. Activation of the erector spinae and multifidus for prolonged period of time (e.g., > 20 minutes) during the arm- and leg-ergometer exercises is a potentially suitable rehabilitation therapy for patients with Parkinson's disease. However, it remains unclear whether activation of these two muscles depends on increased workload during arm- and leg-ergometer exercises even in young healthy persons. 
The purpose of this study was to evaluate the activities of the erector spinae and multifidus during the arm- and leg-ergometer exercises at the same absolute workload in young able-bodied individuals, while recording sEMG. The study was based on the hypothesis that sEMG increases with increased workload and that such increase was similar between the arm- and leg-ergometer exercises.

\section{Materials And Methods \\ Participants}

Fifteen healthy male subjects aged $20 \sim 35$ years were recruited for this study. Their physical characteristics are summarized in Table 1. The study was conducted in accordance with the Declaration of Helsinki. Both the study protocol and consent forms were approved by the Human Ethics Committee. Informed consent was obtained from all the participants by a clear statement including the purpose and risks of the study. The exclusion criteria were acute or chronic low back pain, spinal disease after surgery, acute or chronic cardiovascular, respiratory, neurological, or endocrine disease. No participants met the criteria.

Table 1

Anthropometric characteristics of the study subjects.

\begin{tabular}{|l|ll|}
\cline { 2 - 3 } & $\mathbf{n}$ & $\mathbf{1 4}$ \\
\hline Age (years) & $27.1(3.9)$ \\
\hline Height $(\mathrm{cm})$ & $171.2(5.1)$ \\
\hline Weight $(\mathrm{kg})$ & $62.4(6.9)$ \\
\hline BMI $\left(\mathrm{kg} / \mathrm{m}^{2}\right)$ & $21.3(2.4)$ \\
\hline \multirow{2}{*}{ Exalues are means (SD). BMI, body mass index } \\
\hline \multirow{2}{*}{ Experimental design }
\end{tabular}

All participants exercised on arm- (915E, Monark, Varberg, Sweden) and leg-ergometers (881E, Monark) on the same day from around 6:00 PM. The order of the arm- and leg- ergometer exercises was randomly assigned in each participant. Before the measurement, the protocol was explained to the participant, and a pair of silver-silver chloride cup electrodes for sEMG and another for the electrocardiogram (ECG; Bedside monitor, BSM-2401, Nihon Kohden, Tokyo) were placed on the muscle of interest and on the chest, respectively. The participant sat on either a chair or the leg-ergometer, and after confirming a stable heart rate (HR) on the ECG, the recording of the sEMG was started. After the first exercise session, the participant rested for $\sim 15 \mathrm{~min}$ until HR returned to pre-exercise rate. Then the participant was invited to do another type of ergometer exercise. The study protocol was completed in roughly about one hour.

\section{Arm- and leg-ergometer exercises}


Measurements were conducted at rest over 1-min period while the participant was resting on the chair or the ergometer, gripping the handle bars and the foot on the pedals, respectively. The participant started 1min exercise at 50W and then workload was increased to $100 \mathrm{~W}$ for another $1 \mathrm{~min}$.

\section{Measurements}

sEMGs: A pair of silver-silver chloride surface electrodes was placed on the right side of the erector spinae, multifidus, deltoid, biceps brachii, trapezius, vastus lateralis, and gluteus medius after the respective skin areas were shaved and cleaned with an alcohol swab. The diameter of the recording electrode was $10 \mathrm{~mm}$, and the two electrodes were $2 \mathrm{~cm}$ apart on each site. sEMG of the erector spinae and multifidus were measured in all participants. After measurements were performed in 7 participants, the other 5 sites were added to the measurements, i.e., sEMG signals on 7 sites were recorded in the remaining 7 participants. The electrode positioning on the selected muscles was based on that followed by the SENIAM project [22]. Specifically, the erector spinae: the lateral aspect of the bicuspid process from the L1 spinous process in cephalad direction, multifidus: aligned with a line from the caudal tip posterior spina iliaca superior to the interspace between L1 and L2 interspace at the level of L5 spinous process, deltoid: distal to the anterior acromion on a line connecting the acromion and the thumb, biceps brachii: the midpoint between the acromion and distal 1/3 of the line of the elbow fossa, trapezius: the cross point where a perpendicular line from midpoint between Th3 and the medial edge of the scapula and the line intersecting the acromion and Th5, vastus lateralis: 2/3 from the lateral side of the patella on the line connecting to the superior anterior iliac spine, gluteus medius: the midpoint of the line connecting the iliac crest and the greater trochanter.

The sEMG activities were recorded during the arm- and leg-ergometer exercises using a cordless electromyography system (MQ air, Kissei Comtec, Matsumoto, Japan). The signals were bandpass filtered (8-500 Hz), digitized using an A/D converter (AIO-163202FX-USB, CONTEC, Osaka, Japan), then stored on a computer using a recording software (Vital Recorder, Kissei Comtec) at $1000 \mathrm{~Hz}$ sampling rate.

For ECG recording, the electrodes were placed on the anterior chest and ECG was monitored throughout the study. HR was recorded at the end of each stage of the protocol; at rest and exercise at 50W and 100W. In all participants, HR returned to the baseline level before starting the next session.

\section{Data analysis}

\section{Root mean square (RMS)}

RMS was obtained offline using the digitized stored sEMG signals. To calculate RMS, only the signal recorded between 20.4 to 44.4 s of each workload was selected for each participant because this period was most stable and with minimum artifacts. For each load, the RMS was averaged at rest, 50W, and $100 \mathrm{~W}$ in each participant. Changes $(\Delta)$ from the resting values were calculated at $50 \mathrm{~W}$ and $100 \mathrm{~W}$.

\section{Time series analysis of all recorded muscles}


This analysis was performed on all sEMG recordings in 7 participants during $50 \mathrm{~W}$ exercises. Since cadence was set at $50 \mathrm{rpm}$ in this study, 16 cycles (duration of $1.2 \mathrm{~s}$ ) were included in the selected 20-s data set. We determined the start (time $=0$ ) of each cycle as the onset of activity of the biceps brachii and the vastus lateralis for arm- and leg-ergometers, respectively. In the arm-ergometer exercise, the right handle bar corresponded to 3 o'clock position at $0 \%$ of the one-cycle time series. In the leg ergometerexercise, the right pedal at 12 o'clock position corresponded to $0 \%$ of the one-cycle time series. After normalization of each cycle length as $100 \%$, the RMS of each cycle was summed for every $10 \%$ intervals. Then the 16 cycles were averaged for each workload (Fig. 1). The 10-point data were obtained for each muscle of each participant then all values were normalized as the maximum value in each time series data. The minimum value was calculated from the normalized data. The onset thresholds were defined as $20 \%$ of the determined maximum activity of RMS of each cycle [23].

\section{Statistical analysis}

Two-way ANOVA for repeated measurements was used for analysis of the RMS of the erector spinae and multifidus and HR during arm- and leg-ergometer exercises. The Tukey-Kramer test was used to evaluate the differences between the arm and leg exercises and between $50 \mathrm{~W}$ or $100 \mathrm{~W}$ and rest as post-hoc tests. To analyze the time series of each muscle activity, Tukey-Kramer test was also used to detect significant muscle activities from the minimum value during the time series data of the arm- and leg-ergometer exercises at 50W. All values are expressed as mean (SD) unless otherwise stated. Statistical significance was set at $P<0.05$. All data were analyzed using GraphPad Prism 7 (San Diego, CA).

\section{Results}

The study participants were 14 healthy young men aged 20-35 years. One person was excluded due to a mechanical trouble in the equipment on the day of the test.

\section{RMS of erector spinae during arm- and leg-ergometer exercises (Fig. 2)}

As shown in Fig. 2, In the arm-ergometer exercise, the RMS values of the erector spinae were 0.018 (0.011), $0.026(0.010)$, and $0.041(0.016) \mathrm{mV} / \mathrm{s}$ at rest, $50 \mathrm{~W}$, and $100 \mathrm{~W}$, respectively. The respective RMS values during the leg-ergometer exercise were $0.010(0.007), 0.017(0.008)$, and $0.027(0.010) \mathrm{mV} / \mathrm{s}$. The RMS values were significantly higher in the arm- than the leg-ergometer exercise $(P<0.01)$. The RMS values in both the arm- and leg-ergometers increased with increases in workload (50W and $100 \mathrm{~W}$ vs. rest, $P<0.01 ; 100 W$ vs. 50W, $P<0.01$, each), though the increases in RMSs were comparable $(P>0.05)$.

\section{RMS of multifidus during arm- and leg-ergometer exercises (Fig. 3)}


The RMS values of the multifidus during the arm-ergometer exercise were $0.012(0.007), 0.024$ (0.013), and $0.030(0.016) \mathrm{mV} / \mathrm{s}$ at rest, $50 \mathrm{~W}$ and $100 \mathrm{~W}$, respectively, which were both significantly higher than that at rest $(P<0.01$, each; Fig. 3$)$. Furthermore, the RMS value at $100 \mathrm{~W}$ was comparable to that at $50 \mathrm{~W}$ $(P=0.148)$. In the leg-ergometer exercise, the RMSs of the multifidus at rest was $0.006(0.004) \mathrm{mV} / \mathrm{s}$ and increased to $0.014(0.010)$ and $0.023(0.013) \mathrm{mV} / \mathrm{s}$ at $50 \mathrm{~W}$ and $100 \mathrm{~W}$, respectively. The RMS values increased with increases in workload (50W and $100 \mathrm{~W}$ vs. rest, $\mathrm{P}<0.01 ; 100 \mathrm{~W}$ vs. $50 \mathrm{~W}, \mathrm{P}<0.01$, all). However, the increases in RMSs during arm- and leg-ergometry were comparable $(P>0.05)$.

\section{HR during arm- and leg-ergometer exercises (Fig. 4)}

In the arm-ergometer exercise, the HR values of the erector spinae were 73 (8), 109 (14), and 141 (13) beats/min at rest, $50 \mathrm{~W}$, and $100 \mathrm{~W}$, respectively. The respective HR values of the erector spinae during the leg-ergometer exercise were 77 (5), 108 (16), and 124 (19) beats/min. HR increased with an increase in workload in both ergometer exercises (50W and $100 \mathrm{~W}$ vs. rest, $\mathrm{P}<0.01 ; 100 \mathrm{~W}$ vs. $50 \mathrm{~W}, \mathrm{P}<0.01$, all). $\Delta \mathrm{HR}$ at $50 \mathrm{~W}$ in the arm and leg exercises were similar, but $\triangle \mathrm{HR}$ at $100 \mathrm{~W}$ was higher in the arm exercise than leg exercise $(P<0.01$; Fig. 4).

\section{Time series of one-cycle \%RMSs for each muscle during exercise (Fig. 5)}

As shown in Fig. 5, The biceps brachii was active from 0 to $30 \%$ mainly related to elbow flexion and from 90 to $100 \%$ partially related with supination. The erector spinae was active from 70 to $100 \%$ and from 0 to $20 \%$, i.e., almost the same time as the biceps were active. On the other hand, the multifidus was active from 40 to $90 \%$, mostly during the pushing phase. The gluteus medius was active during almost the same phase as the multifidus (30 to $90 \%$ ). The trapezius and deltoid were active from 0 to $40 \%$ and 20 to $50 \%$, respectively. No activity was detected in the vastus lateralis during the test.

The vastus lateralis was active from 0 to $40 \%$ mainly in relation with knee extension and from 90 to $100 \%$ in relation with slight hip flexion. Both the erector spinae and multifidus were active from 40 to $70 \%$. The active phase of the gluteus medius almost overlapped; from 20 to $40 \%$. Minor activities were observed in the biceps brachii, deltoid, and trapezius.

\section{Discussion}

This is the first study to show increases in activities of the erector spinae and multifidus during arm- and leg-ergometer exercises with increased workload. The results suggest that although the metabolic responses were higher in the arm exercise relative to the leg exercise, the activities of the paraspinal muscles were similar in both exercises. Furthermore, the timing of activation of the erector spinae was almost similar to that of the ipsilateral biceps, i.e., agonist muscles, and that of the multifidus was activated oppositely during arm-ergometer exercise, suggesting that these paraspinal muscles increased their activities to support rotational moment of the trunk. The activation time windows of the two paraspinal muscles were opposite to those of the ipsilateral vastus lateralis during the leg-ergometer 
exercise, suggesting that these paraspinal muscles increased their activities to prevent the lateral bending moment of the trunk on the tilted side.

Our results showed differences in resting muscle activities of the erector spinae muscle with other muscles (Fig. 2), while the increases in SEMG signals of the erector spinae and multifidus were comparable during arm- and leg-ergometer exercises. Previous studies showed that the activity of the erector spinae changes with the angle of pelvic tilt, and this may be the reason for the difference at rest in the present study [24-25].

In the arm-ergometer exercise, the trunk rotated during pulling an object with one arm, and the activity of the ipsilateral erector spinae increased in order to increase the rotation moment, which was similar to the observation reported by Lavender et al. [26]. In addition, the multifidus is reported to support contralateral trunk rotation [27]. The above previous findings suggest that the ipsilateral erector spinae and the contralateral multifidus were active because of slight trunk rotation during pulling the handle with ipsilateral arm. In the leg-ergometer exercise, tilting of the seated person on coronal plane was associated with the appearance of lateral bending moment of the trunk on the tilted side, and activation of the contralateral erector spinae and multifidus, which allowed stabilization of the trunk [28]. The ipsilateral bending moment of the trunk seemed to have occurred during stepping on the pedal with one leg in the present study. The results suggest that simultaneous contractions of the contralateral erector spinae and multifidus during the leg-ergometer exercise opposed the ipsilateral bending moment of the trunk, thus ensuring maintenance of trunk balance. Furthermore, the strength of the erector spinae and multifidus seems to be depended on the magnitude of the rotation moment and of the lateral bending moment due to the increase in workload during the arm- and leg-ergometer exercises, respectively.

Also, in agreement with previously data [29], our results showed increased HR and oxygen uptake with the increased workload during the arm- and leg-ergometer exercises, but the increases in these two parameters at any given increase in workload were higher during the arm-ergometer exercise than the legergometer exercise [29]. Thus, the present study suggests that the stress on the erector spinae and multifidus themselves during the arm-ergometer exercise is similar to that during the leg-ergometer exercise, although the energy efficiency is worse during arm-ergometer exercise compared with to legergometer exercises.

\section{Limitations}

The present study did not include motion analysis during the two forms of exercises. One-minute submaximal arm-ergometer exercise in healthy participants was reported to be accompanied by trunk rotation [30]. Also, anterior-posterior changes in the center of gravity were described during 30 minutes of arm- and leg-ergometer exercises at $50 \%$ of maximum [31]. Trunk movement similar to that mentioned above could have occurred in the present study, though this is speculative at present.

The present participants were able to complete the ergometer exercise at 50W and 100W, workloads, which are relatively high for frail elderly subjects. Furthermore, the present study involved only 2 minutes 
of exercise, whereas the recommended training time for aerobic exercise in patients with Parkinson's disease is a minimum of $20 \mathrm{~min}$, for example [22]. Therefore, the effect of prolonged or repeat arm- and leg-ergometer exercise on paraspinal muscles remains unclear.

\section{Perspectives}

Patients with Parkinson's disease experience more falls than age-matched healthy subjects [32] and have shown higher baseline levels of paraspinal muscle activity during walking, which is attributed to reduced muscle strength [19]. In such patients, exercise-based interventions can improve motor dysfunction and imbalance [33], which cannot be controlled by pharmacological and surgical interventions [34]. Current rehabilitation recommendations to improve posture in these patients include core trainings, which promote activities of trunk muscles [35]. Because prolonged repeated contractions of the erector spinae and multifidus involuntarily would be more beneficial for the patients than just performing core-building trainings, we think that a prospective study is needed to assess the effects of arm- and leg-ergometer exercises in elderly persons and/or patients with Parkinson's disease.

\section{Conclusions}

Arm- and leg-ergometer exercises were associated with increased sEMG activities of the erector spinae and multifidus and such increase was proportionate with increases in workload. The increases in activities were similar in the arm- and leg-ergometer exercises.

\section{Abbreviations}

sEMG: surface electromyogram

MVC: maximal voluntary contraction

ECG: electrocardiogram

HR: heart rate

RMS: Root mean square

\section{Declarations}

\section{Ethical Approval and consent to participate}

The study protocol and consent forms were approved by the Human Ethics Committee of Wakayama Medical University (approval \#2609).

\section{Consent for publication}

Not applicable 
Availability of data and materials

The dataset supporting the conclusions of this article is available from the corresponding author on reasonable request.

\section{Competing interests}

The authors have no conflicts of interest directly relevant to the content of this article.

\section{Funding}

This work was supported by a grant from Nachikatsuura Research Foundation (\#L1221) to F.T. and Grants-in-Aid for Scientific Research 21K11364 to Y-i.K.

\section{Authors' contributions}

D.S., T.H., T.Y. and F.T. conceived and designed the research protocol; D.S., Y.M., and T.H. completed the data collection; D.S., T.H. T.Y., and Y-i.K. analyzed the data; D.S., T.H., Y-i.K., and F.T. interpreted the results; D.S. prepared the figures; D.S., T.H. and Y-i.K. drafted the manuscript; all authors read, commented and revised the first draft; and then approved the final version of the manuscript.

\section{Acknowledgments}

The authors gratefully thank the all participants.

\section{References}

1. Nara, I. et al. Standard Physical Therapy, Occupational Therapy Specialized Foundation Field. Anatomy (Institute of Medicine, Tokyo, 2012).

2. Silva, R. A. et al. Age and low back pain related differences in trunk muscle activation during onelegged stance balance task. Gait Posture, 69, 25-30 (2019).

3. Aruin, A. S. \& Latash, M. L. Directional specificity of postural muscles in feed-forward postural reactions during fast voluntary arm movements. Exp Brain Res, 103, 323-332 (1995).

4. Chiou, S. Y., Gottardi, S. E., Hodges, P. W. \& Strutton, P. H. Corticospinal excitability of trunk muscles during different postural tasks. PLOS ONE, 11, e0147650 (2016).

5. Bussey, M. D., Castro, M. P., Aldabe, D. \& Shemmell, J. Sex differences in anticipatory postural adjustments during rapid single leg lift. Hum Mov Sci, 57, 417-425 (2018).

6. Cavallari, P., Bolzoni, F., Bruttini, C. \& Esposti, R. The organization and control of intra-limb anticipatory postural adjustments and their role in movement performance. Front Hum Neurosci, 10, 525 (2016).

7. Flueck, J. L. Experimental protocol of a three-minute, all-out arm crank exercise test in spinal-cord injured and able-bodied individuals. J Vis Exp, 124, 55485 (2017). 
8. Campbell, E., Coulter, E. H. \& Paul, L. High intensity interval training for people with multiple sclerosis: A systematic review. Mult Scler Relat Disord, 24, 55-63 (2018).

9. Francois, M. E. et al. Similar metabolic response to lower- versus upper-body interval exercise or endurance exercise. Metabolism, 68, 1-10 (2017).

10. Marks, C. R. C., Dupuie, L. \& Patros, J. Stability ball sitting elevates peak arm ergometry oxygen consumption and heart rate. Int J Exerc Sci, 5, 360-366 (2012).

11. Keyser, R. E., Mor, D. \& Andres, F. F. Cardiovascular responses and anaerobic threshold for bicycle and arm ergometer exercise. Arch Phys Med Rehabil, 70, 687-691 (1989).

12. Bouaziz, W., Schmitt, E., Kaltenbach, G., Geny, B. \& Vogel, T. Health benefits of cycle ergometer training for older adults over 70: a review. Eur Rev Aging Phys Act, 12, 8 (2015).

13. Xiao, J., Gao, J., Wang, H. \& Yang, X. The sEMG characteristics of the low back muscles during aerobic cycling. Biomed Mater Eng, 24, 2571-2576 (2014).

14. Marks, C. R. C., Hylland, K. E. \& Terrell, J. Stability ball sitting versus chair sitting during sub-maximal arm ergometry. Int J Exerc Sci, 5, 16-25 (2012).

15. Oliveira, A. S. \& Gonçalves, M. Lumbar muscles recruitment during resistance exercise for upper limbs. J Electromyogr Kinesiol, 19, 737-745 (2009).

16. Clark, D. R., Lambert, M. I. \& Hunter, A. M. Trunk muscle activation in the back and hack squat at the same relative loads. J Strength Cond Res, 33, 60-69 (2019).

17. Alemi, M. M., Geissinger, J., Simon, A. A., Chang, S. E. \& Asbeck, A. T. A passive exoskeleton reduces peak and mean EMG during symmetric and asymmetric lifting. $J$ Electromyogr Kinesiol, 47, 25-34 (2019).

18. Murata, K. et al. Clinico-pathological study of erector spinae muscles in patients with Parkinson's disease., 21, 13347 (2010).

19. Margraf, N. G., Rogalski, M., Deuschl, G. \& Kuhtz-Buschbeck, J. P. Trunk muscle activation pattern in parkinsonian camptocormia as revealed with surface electromyography. Parkinsonism Relat Disord, 44, 44-50 (2017).

20. Margraf, N. G. et al. MRI of lumbar trunk muscles in patients with Parkinson's disease and camptocormia. J Neurol, 262, 1655-1664 (2015).

21. Nobel, M., Millholen, A. \& Kelley, S. ACSM's Guidelines for Exercise Testing and Prescription 10th edn (Wolters Kluwer, South Holland, 2018).

22. Hermens, H. J., Freriks, B., Disselhorst-Klug, C. \& Rau, G. Development of recommendations for SEMG sensors and sensor placement procedures. J Electromyogr Kinesiol, 10, 361-374 (2000).

23. Silva, J. C. L. et al. Quadriceps and hamstring muscle activity during cycling as measured with intramuscular electromyography. Eur J Appl Physiol, 116, 1807-1817 (2016).

24. Azghani, M. R., Nazari, J., Sozapoor, N., Jafarabadi, M. A. \& Oskouei, A. E. Myoelectric activity of individual lumbar erector spinae muscles variation by differing seat pan depth. Int J Occup Environ Med, 10, 137-144 (2019). 
25. Sullivan, P. B. O. et al. The effect of different standing and sitting postures on trunk muscle activity in a pain-free population. Spine, 27, 1238-1244 (2002).

26. Lavender, S. A., Chen, S. H., Li, Y. C. \& Andersson, G. B. Trunk muscle use during pulling tasks: effects of a lifting belt and footing conditions. Hum. Factors, 40, 159-172 (1998).

27. Kawakami, K. \& Isogai, K. Anatomy and surface anatomy of muscles second edition (Taihoukaku, Kanagawa, 2013).

28. Kuster, R. P., Bauer, C. M. \& Baumgartner, D. Is active sitting on a dynamic office chair controlled by the trunk muscles? PLoS One, 30, e0242854 (2020).

29. Eston, R. G. \& Brodie, D. A. Responses to arm and leg ergometry. Br J Sports Med, 20, 4-6 (1986).

30. Faupin, A., Gorce, P. \& Meyer, C. Effects of type and mode of propulsion on hand-cycling biomechanics in nondisabled participants. J Rehabil Res Dev, 48, 1049-1060 (2011).

31. Hill, M. W., Sampson, M. G., Duncan, M. L. \& Price, M. J. The effects of maximal and submaximal arm crank ergometry and cycle ergometry on postural sway. Eur J Sport Sci, 14, 782-790 (2014).

32. Cole, M. H., Naughton, G. A. \& Silburn, P. A. Neuromuscular impairments are associated with impaired head and trunk stability during gait in Parkinson fallers. Neurorehabil Neural Repair, 31, 34-47 (2017).

33. Hubble, R. P., Naughton, G., Silburn, P. A. \& Cole, M. H. Trunk exercises improve gait symmetry in Parkinson disease: A blind phase II randomized controlled trial. Am J Phys Med Rehabil, 97, 151159 (2018).

34. Bloem, B. R., Hausdorff, J. M., Visser, J. E. \& Giladi, N. Falls and freezing of gait in Parkinson's disease: a review of two interconnected, episodic phenomena. Mov Disord, 19, 871-884 (2004).

35. Smania, N. et al. Effect of balance training on postural instability in patients with idiopathic Parkinson's disease. Neurorehabil Neural Repair, 24, 826-834 (2010).

\section{Figures}




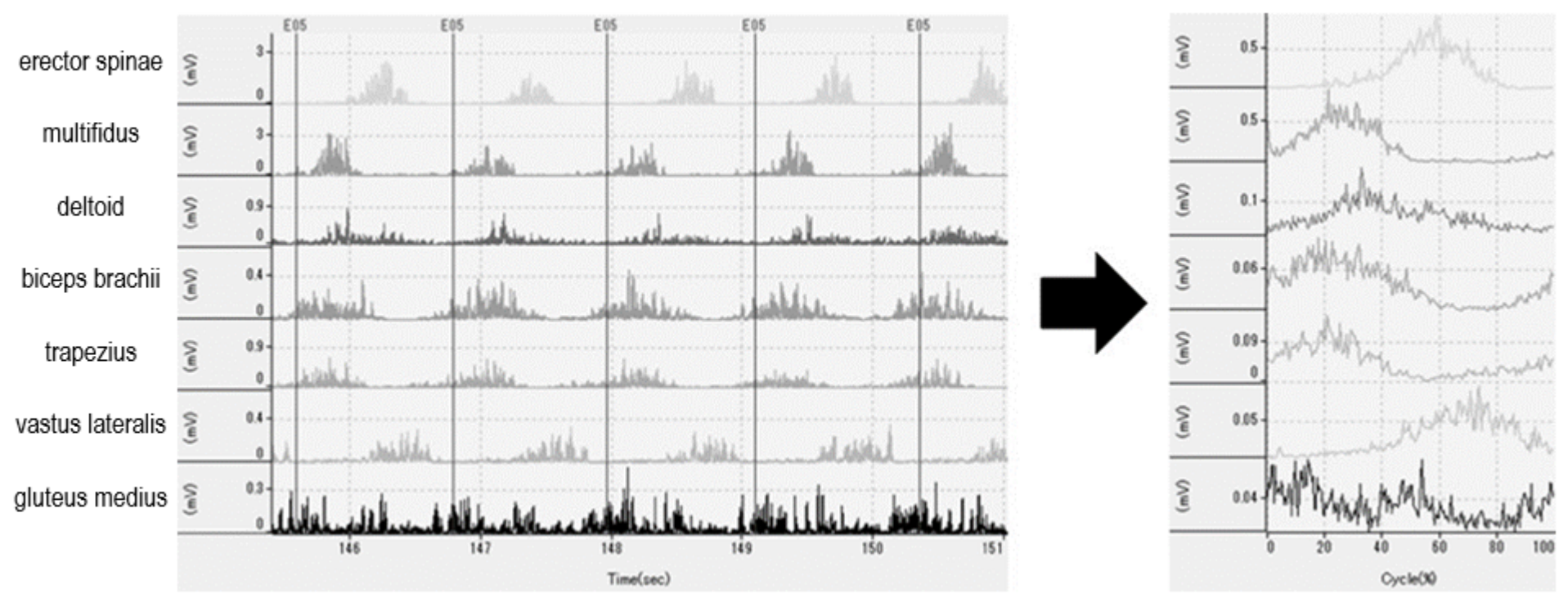

\section{Figure 1}

A typical example which shows a method used for time series analysis of surface electromyogram. Surface electromyograms (sEMG) on the right side of the erector spinae, multifidus, deltoid, biceps brachii, trapezius, vastus lateralis, and gluteus medius during arm or leg exercises at 50W are shown in a participant. Since cadence was set at $50 \mathrm{rpm}$ in this study, 16 cycles (duration of 1.2s) were included in the selected data set for around 20s. All sEMG are rectified (left panel). Then the onset of activity of the biceps brachii (the right handle bar corresponded to 3 o'clock position) and the vastus lateralis (the right pedal at 12 o'clock position) for arm- and leg-ergometers, respectively, are determined as the start (time $=0$ ) of each cycle, and the 16 cycles were averaged after normalization of each cycle length as $100 \%$ (right panel).
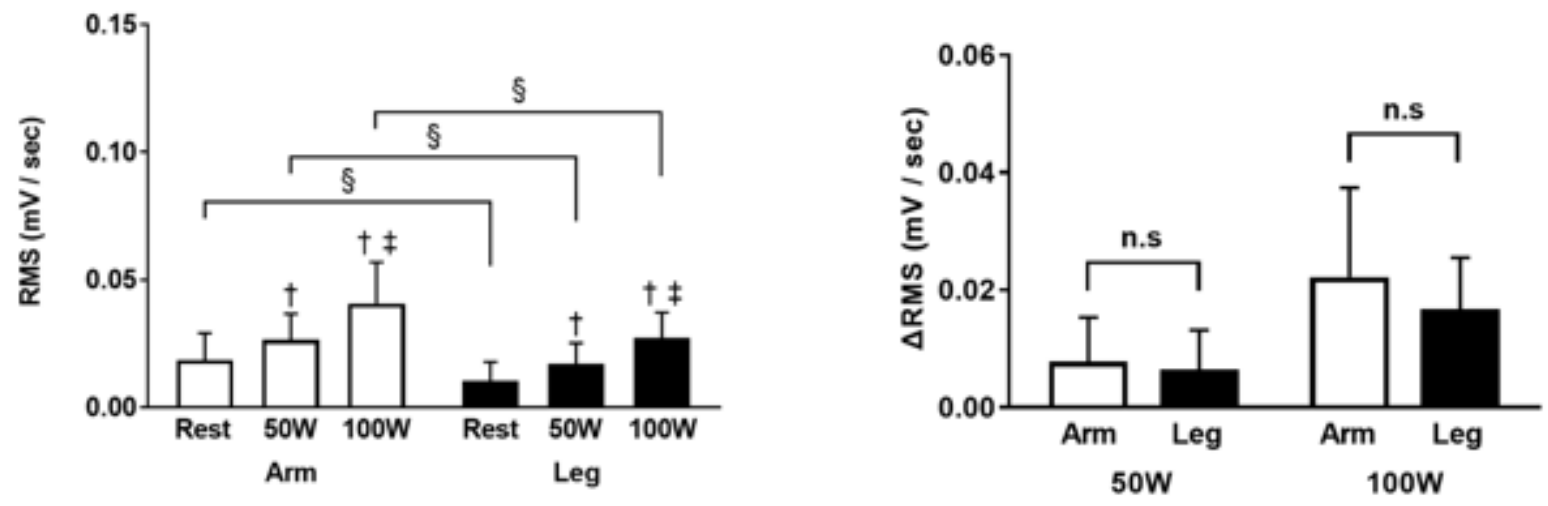

Figure 2

RMS of the erector spinae during arm- and leg-ergometer exercises. Left) Values of root mean square (RMS) at rest and at 50W and $100 \mathrm{~W}$ of exercise. Right) Changes $(\Delta)$ from the baseline (Rest). Values are 
mean \pm SD for 14 subjects. $\dagger, \ddagger$, compared with Rest and 50W, respectively; $\S$, different from that during the arm exercise at the level of $\mathrm{P}<0.05$. n.s, not significant.
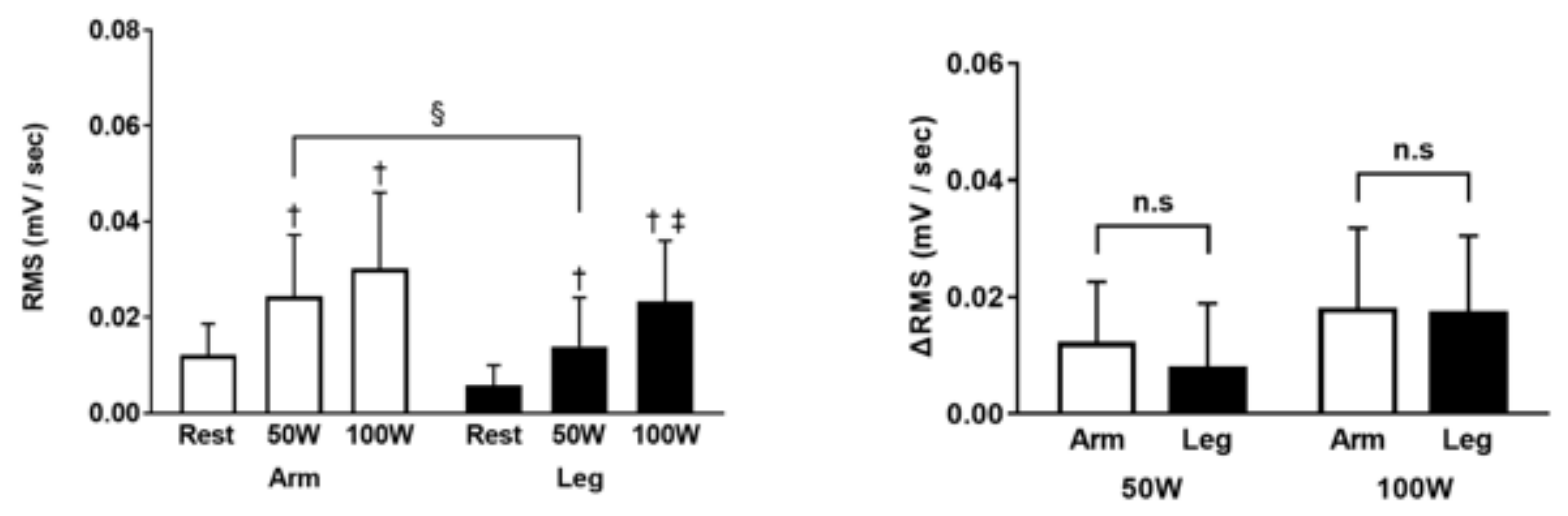

\section{Figure 3}

RMS of the multifidus muscle during arm- and leg-ergometer exercises. Left) Values of root mean square (RMS) at rest and at $50 \mathrm{~W}$ and $100 \mathrm{~W}$ of exercise. Right) Changes $(\Delta)$ from the baseline (Rest). Values are mean $\pm S D$ for 14 subjects. $\dagger, \ddagger$, compared with Rest and $50 \mathrm{~W}$, respectively; $\S$, different from that during the arm exercise at the level of $P<0.05$. n.s, not significant.
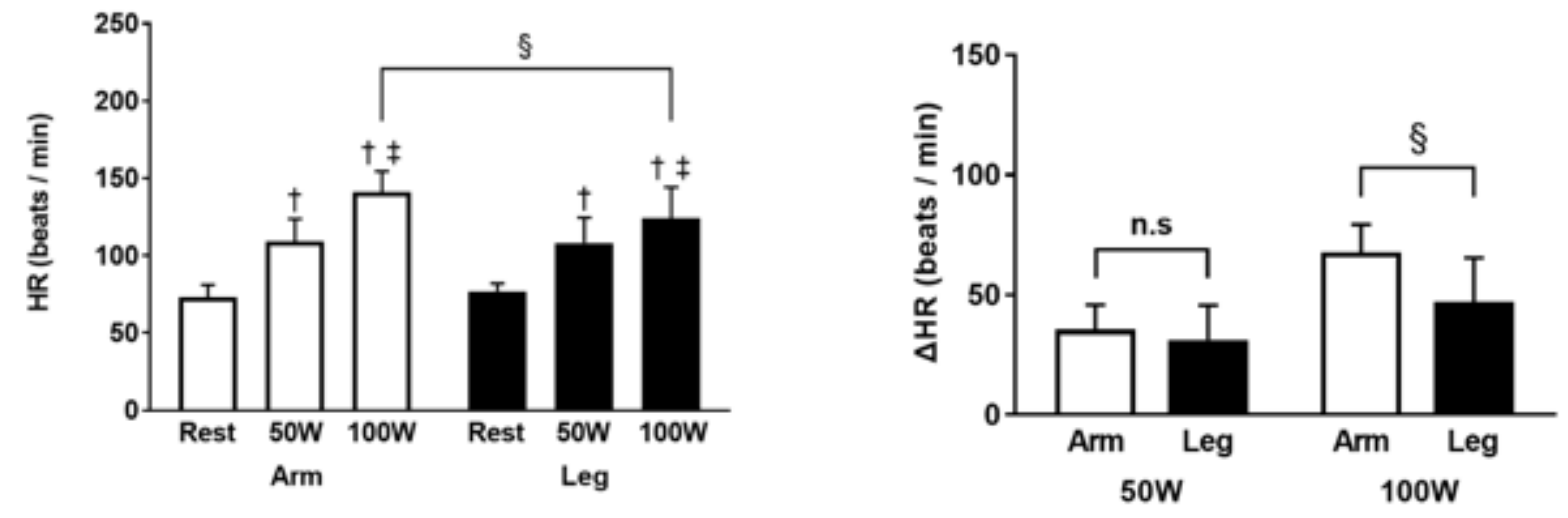

Figure 4

HR of the erector spinae during arm- and leg-ergometer exercises. Left) Heart rate (HR) at rest and at 50W and $100 \mathrm{~W}$ of exercise. Right) Changes $(\Delta)$ from the baseline (Rest). Values are mean $\pm S D$ for 14 subjects. $\dagger, \ddagger$, compared with Rest and 50W, respectively; §, different from that during the arm exercise at the level of $\mathrm{P}<0.05$. 


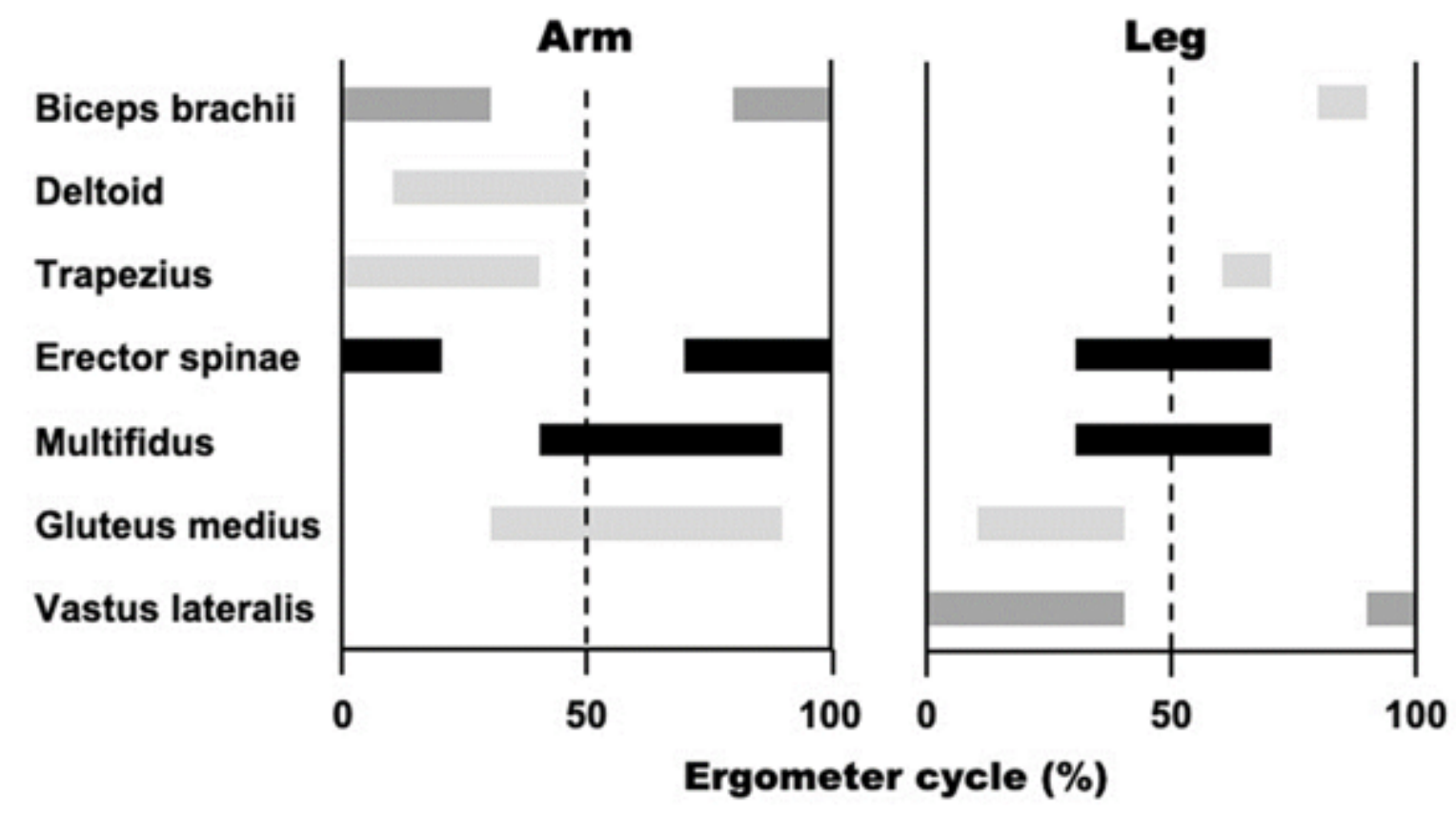

Figure 5

Time windows of each sEMG activity during one cycle of arm and leg ergometer exercises. Data were obtained from 20 to $40 \mathrm{sec}$ at $50 \mathrm{~W}$ in both exercises $(n=7)$. Sixteen cycles (duration of $1.2 \mathrm{~s})$ were included in the selected 20-s data set. The start of each cycle as the onset of activity of the biceps brachii and the vastus lateralis for arm and leg ergometers represented time $=0$. The onset thresholds were defined as $20 \%$ of the determined maximum activity of RMS every $1 \mathrm{~s} 26)$. The time series of the onset and end of each cycle were defined as $0 \%$ and $100 \%$, respectively. The RMS values were averaged every $10 \%$ of each cycle. The 10-points data were obtained from each muscle of each participant then all values were expressed relative to the maximum value in each time series data. The minimum value was calculated from the normalized data. 